\title{
Remote and Feedback Control of the Flap Angle in a Wind Tunnel Test Model by Optical Measurement
}

\author{
Kazuhisa Chiba ${ }^{1, * \mathbb{D}}$, Tatsuro Komatsu ${ }^{1}$, Hiroyuki Kato ${ }^{2}$ and Kazuyuki Nakakita ${ }^{2}$ \\ 1 The University of Electro-Communications, 1-5-1, Chofugaoka, Chofu, Tokyo 182-8585, Japan; \\ koma20228@gmail.com \\ 2 Japan Aerospace Exploration Agency (JAXA), 7-44-1, Jindaiji-Higashi, Chofu, Tokyo 182-8522, Japan; \\ kato.hiroyuki@jaxa.jp (H.K.); nakakita.kazuyuki@jaxa.jp (K.N.) \\ * Correspondence: kazchiba@uec.ac.jp; Tel.: +81-42-443-5420
}

Received: 28 November 2019; Accepted: 29 January 2020; Published: 31 January 2020

\begin{abstract}
We have developed a remote and precise feedback control system using optical measurement technology to alter the angle of a flap, which is part of a wind tunnel test model, automatically and to earn the aerodynamic data efficiently. To rectify the wasteful circumstance that Japan Aerospace Exploration Agency (JAXA)'s low-turbulence wind tunnel stops ventilation every time to switch model configurations, we repaired hardware for remote operation and generated software for feedback control. As a result, we have accomplished a system that dramatically advances the efficiency of wind tunnel tests. Moreover, the system was able to consider the deformation of the model through optical measurement; the system controlled flap angles with errors less than the minimum resolution of optical measurement equipment. Consequently, we successfully grasped the nonlinearity of three aerodynamic coefficients $C_{L}, C_{D}$, and $C_{M p}$ that was impossible so far.
\end{abstract}

Keywords: remote operation; feedback control; optical measurement; wind tunnel test; aerodynamic characteristics; aircraft; flap

\section{Introduction}

Aircraft wings optimized for cruise conditions provide control devices to ensure aerodynamic performance during takeoff and landing [1]. For safe takeoff and landing, it is important to assess the aerodynamic performance accurately for deploying control devices. Computational fluid dynamics (CFD) technology has made tremendous progress [2-4]; it has advantages in that not only can it reduce capital investment and maintenance costs, but it also can acquire physical quantity at any position in a computational domain. However, to realize accurate aerodynamic performance prediction for control devices, CFD is not mature yet; we deepen the perception of knowledge through the American Institute of Aeronautics and Astronautics high-lift prediction workshops [5-7]. On the other hand, in the wind tunnel test, we can obtain spatial data through particle image velocimetry (PIV) [8] measurements; we can comprehensively gain surface pressure/temperature through the invention and development of Pressure/Temperature Sensitive Paint (PSP/TSP) [9]. In recent years, it has become possible to procure unsteady data, too [10]. Although there are problems such as wall interference of wind tunnels and the Reynolds number effect, we can earn data without any mathematical modeling, so it remains an indispensable means for aerodynamic performance evaluation.

A typical wind tunnel is a system that installs a large actuator outside a measuring chamber and changes the angle of attack of an entire model. Thus, it is necessary to temporarily suspend the ventilation of the wind tunnel to alter only a part of the wind tunnel model like control devices; we adjust the configuration of it by hand. Then, we should recommence ventilation. Because we can ordinarily measure at most five configurations per day, we cannot obtain a sufficient amount of data 
during a limited test period, which is abundantly unproductive. Research and development have been implemented to extend on dynamic testing capabilities that Germany and the Netherlands have advanced for many years to solve this dilemma [11,12]. Airbus has proposed the motorization of wind tunnel test models that can be remotely controlled [13]. The Boeing company has similarly earned patents on the measurement technology of wind tunnel tests in the US [14]. In this way, wind tunnel tests are becoming more efficient, but no papers discuss the particulars of the data acquired from these systems; the exactitude of the data and the usefulness of the method are unknown.

This study has two purposes: the efficiency of wind tunnel tests and highly accurate configuration settings. We remotely manipulated an actuator to change model configurations sequentially without resting ventilation to enhance the efficiency of wind tunnel tests. Actuators used in wind tunnel models have recently exhibited sophisticated performance [15], so we extended a system in which a small actuator is installed in a simple 3D rectangular wing model with a flap so that we manage the flap angle remotely. However, an error inevitably arises between an input value and an actual rotating angle from play due to the actuators' characterization. An error also results from the elastic deformation of wind tunnel models. To minimize these errors is crucial to assure the exactitude of wind tunnel test data. Thus, we fulfilled an automated feedback control of the actuator. We prepared three parameters to utilize for our feedback control and inspect the precision of rotating amounts of flap angles for them. The first parameter is the pulse number of the stepping motors in the actuator; the second is the encoder value of the flap hinge line; the third is the 3D surface coordinates of the wind tunnel test model based on optical measurement. This study adopted the 3D surface coordinates using the optical measurement system $[16,17]$ for the first time to consider the elastic deformation of the model, so we focused on validating its efficacy.

This article is structured as follows. In Section 2, we explain the constructed feedback control system. Section 3 outlines the model and pieces of measurement equipment used in the wind tunnel system. In Section 4, we assess the precision accomplished by the feedback control system using optical measurement. Section 5 concludes this article.

\section{Feedback Control System Using Optical Measurement Technology}

By bringing the actual flap angle closer to the input value as much as possible, we can procure highly accurate aerodynamic data. Thus, this research diverts the optical measurement system that Japan Aerospace Exploration Agency (JAXA) has been studying. Optical measurement is a technique to measure variations in the physical quantity of flow fields and geometrical deformations of wind tunnel test models through optical instruments such as laser and camera. JAXA has been researching the flow field measurement via PIV $[18,19]$ and PSP $[17,20]$ using this system and has recently constructed a hybrid wind tunnel through data assimilation with CFD. We divert a part of the optical measurement system configured to accomplish the purpose of this study and derive actual flap angles from photographed image data of the wind tunnel test model.

Inferring flap angles is based on the principle of triangulation; two cameras measure the 3D coordinates of markers stuck on the model surface. We estimated normal vectors of the coordinate values at each marker position and procured relative flap angles before and after the flap driving. The flow of the derivation is as follows:

1. Camera calibration: Calibration is conducted to define parameters related to camera position and attitude. We employed stereo camera calibration, which is one function in MathWorks ${ }^{\circledR}$, MATLAB's Computer Vision System Toolbox ("Camera Calibration Toolbox for Matlab" available online at http:/ / www.vision.caltech.edu/bouguetj/calib_doc/ [retrieved 30 March 2018]). Three images can estimate parameters required for MathWorks' function, but we have been using at least 50 images this time to diminish errors after calibration.

2. Marker detection: We affixed 15 circular markers on the flap (three in the chordwise direction times five in the spanwise direction) to gain their coordinates. A photographed image detected a circular shape in the prescribed radius range as a marker; we numbered them and computed their 
barycentric coordinates. We mutually collated two sets of the barycentric coordinates calculated from the images taken by the two cameras and derived the 3D coordinates of the markers.

3. Derivation of the flap angle: The 3D coordinate data set of the detected markers formed planes using adjacent three points; we assessed the normal vectors of those planes. We computed the flap angle with the difference from the reference flap angle of 0 degrees. Note that we decomposed normal vectors in three axial directions; we utilized only two vectors orthogonal to the flap hinge line for measuring the flap angle.

$\theta_{\text {optical }}$ denotes the feedback value by optical measurement. Further, the system can make feedback controls using the pulse number of stepping motors $\theta_{\text {pulse }}$ and the encoder values $\theta_{\text {encoder }}$ as parameters.

\section{System Configuration}

Tests were supposed to run at the JAXA $0.65 \mathrm{~m} \times 0.55 \mathrm{~m}$ low-turbulence wind tunnel, which is a closed-circuit type and the wind velocity attains from 5 to $50 \mathrm{~m} / \mathrm{s}$. As we show a simple block diagram in Figure 1, the present system was composed of a wind tunnel test model, an optical measurement system, and a laptop that remotely controls the wind tunnel test model. When we input a target angle of the flap $\theta_{\text {target }}$, the flap rotates to coincide with it. The system curtailed errors of the flap angles within $5.7 \times 10^{-4}$ degrees in prior trials under no wind condition and was independent of $\theta_{\text {target }}$ and amounts of the traverse. We describe the details of the wind tunnel test model, optical measurement equipment, and aerodynamic measurement equipment below.

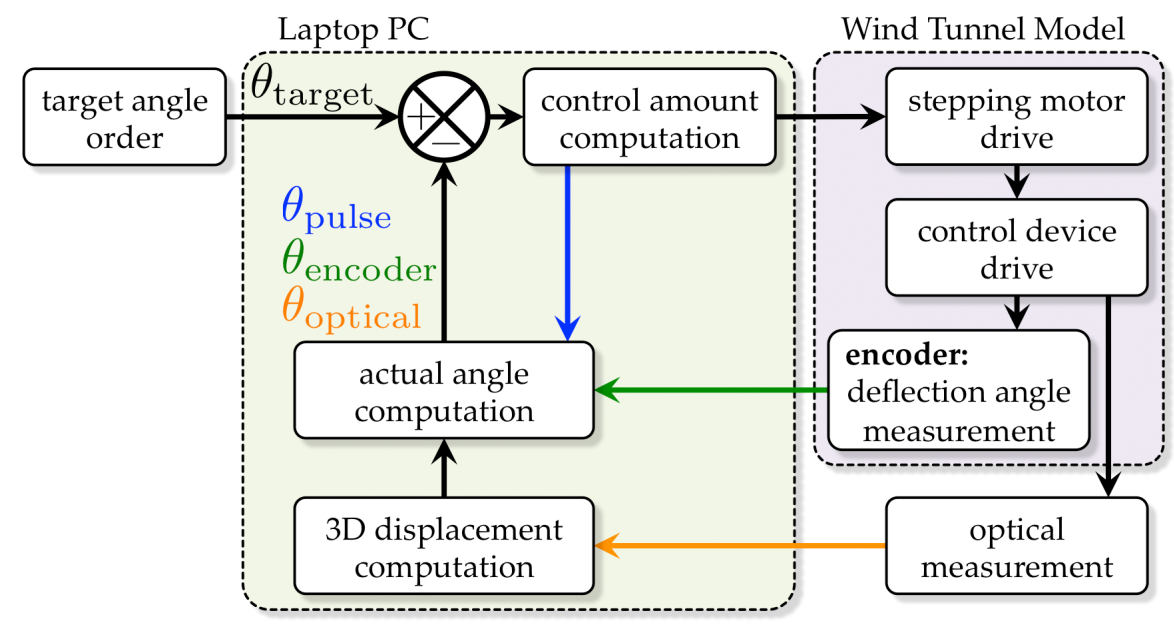

Figure 1. Block diagram of the system configuration.

\subsection{Wind Tunnel Test Model}

We took a commercialized actuator as small as possible and made the wind tunnel test model with a stepping-motor-driven actuator inside it. Since the wing needed thickness suitable to contain the actuator, we chose the NACA0018 airfoil shape. We can alter either both-end or one-end fixed configuration by attaching/detaching the wing tip parts. We used the model with the both-end supported configuration for fixing horizontally in the wind tunnel to perform control performance tests. By contrast, we utilized the one-end supported configuration perpendicularly to the balance in the wind tunnel to measure three components of the aerodynamic force. We designed the model with the dimensions shown in Table 1 according to the size of the wind tunnel test section.

Installed instruments had the following requirement; they need to attain a torque that surmounts the aerodynamic force that the flow of the wind tunnel generates. Even in wind tunnel tests with the maximum flow speed of $50 \mathrm{~m} / \mathrm{s}$, we need to stipulate the lower limit of the required torque because we should select an actuator capable of generating sufficient torque against the aerodynamic force. 
Table 1. The dimensions of the wind tunnel test model.

\begin{tabular}{lr}
\hline Part & Dimensions (mm) \\
\hline Main wing chord length & 250 \\
Main wing span length (configuration supported on one side) & 390 \\
Main wing span length (configuration supported on both sides) & 550 \\
Flap chord length & 50 \\
Flap span length & 200 \\
\hline
\end{tabular}

Figure 2 presents the wind tunnel test model drawn by CAD software and created practically. To satisfy all the aboverequirements, we stored the equipment in the thickest location of the wing and transmitted the torque to the hinge line of the flap using bevel gears and a shaft shown in Figure 3. The details of the installed devices are as follows:

- Stepping motor: ARM24SAK made by Oriental Motor Co., Ltd. (Tokyo, Japan). We selected an actuator driven by two stepping motors. This motor works through a pulse input; the resolution is 0.0036 degrees. The rotation speed is $70 \mathrm{rpm}$. For precise positioning of the flap, the backlash was prevented by rotating the two motors in pairs and rotating in opposite directions for the operating axis each other. The motor adopts harmonic gears; the speed reduction ratio is 100.

- Encoder: MAS-3-4096N1 made by MicroTech Laboratory Inc. (Sagamihara, Kanagawa-prefecture, Japan). To mechanically measure the rotation angle of the operating axis directly, we installed a small encoder whose resolution is the catalog value of 0.088 degrees.

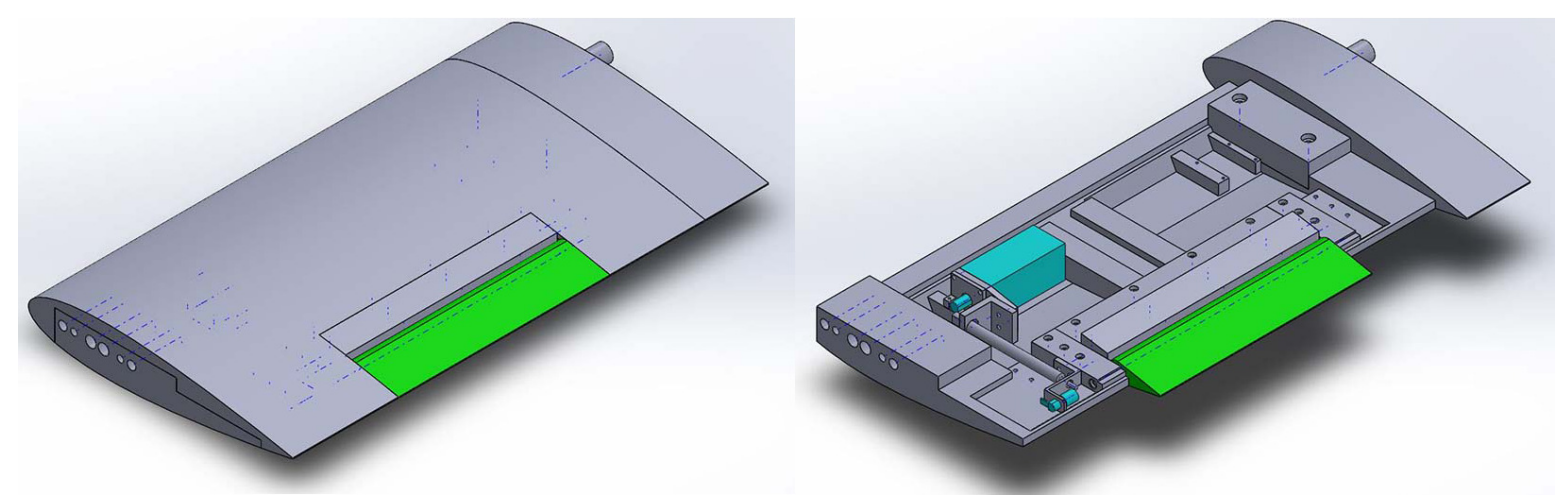

(a) Appearance and internal structure on CAD illustration.

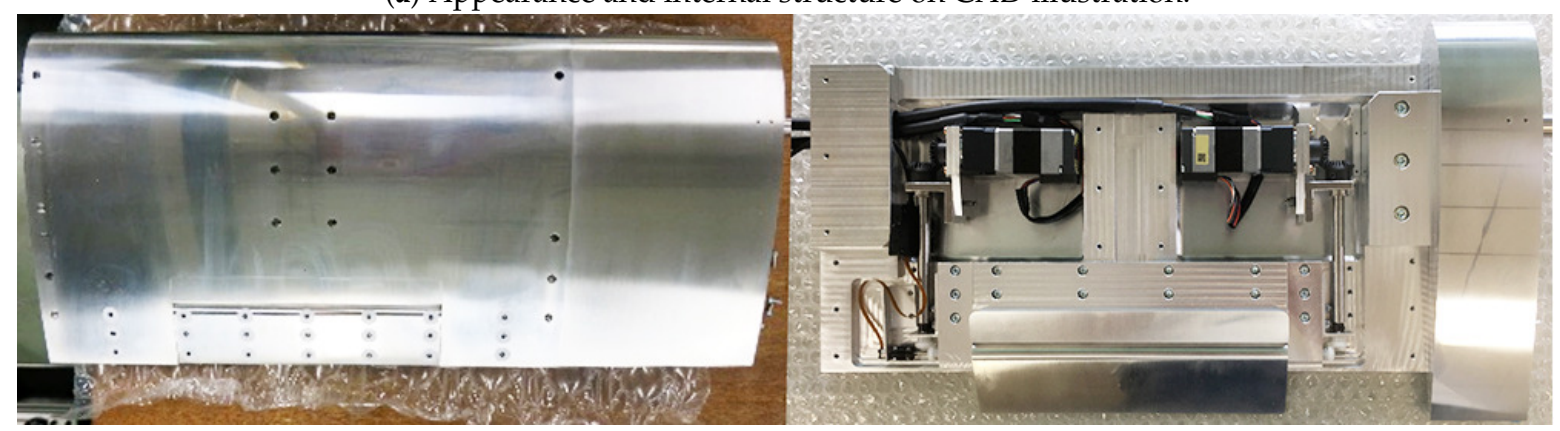

(b) Practically created model and its interior.

Figure 2. NACA0018 wind tunnel test model with the flap. 

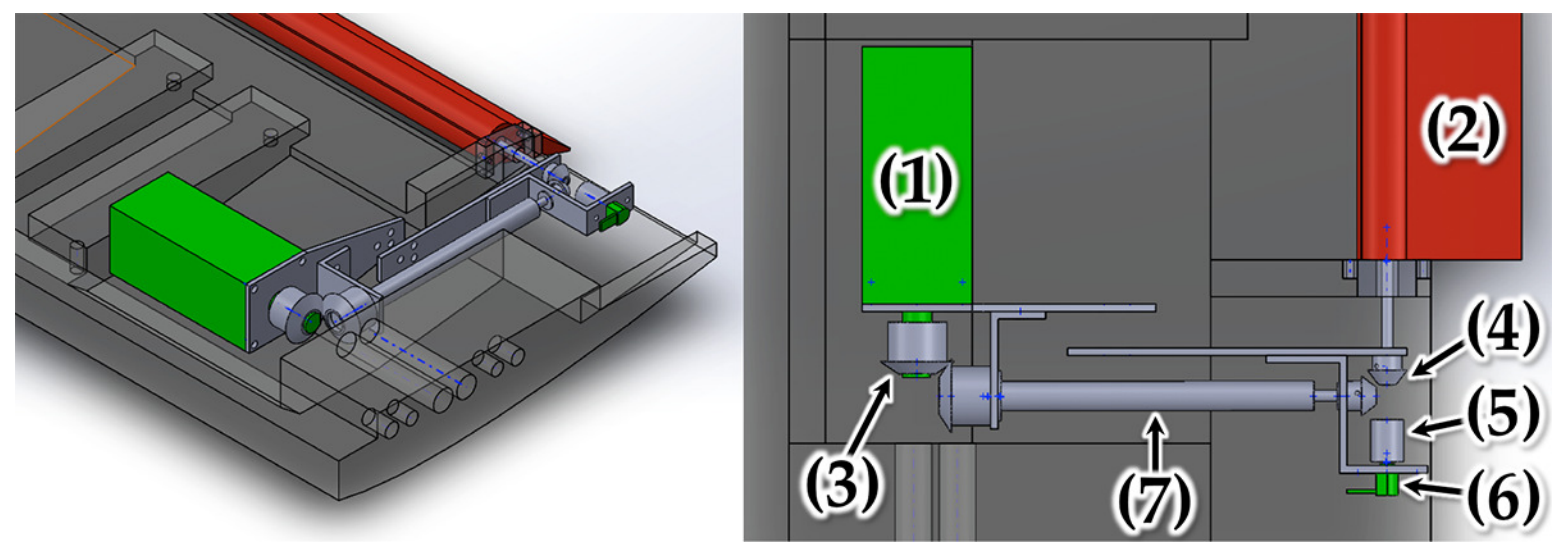

Figure 3. Actuator mechanism; (1) chamber to store stepping motors, (2) flap, (3) bevel gears on the motor side, (4) bevel gears on the flap side, (5) coupling, (6) encoder, and (7) stepped shaft.

3.2. Instruments for Optical Measurement

- Camera: We used Manta G-1236B made by Ad Science Inc. (Funabashi, Chiba-prefecture, Japan). This gigabit ethernet camera has a SONY IMX304 CMOS progressive sensor; the resolution is $4112 \times 3008$ dpi.

- Lighting device: A Xenon light source made by Hamamatsu Photonics K. K. (Hamamatsu, Shizuoka-prefecture, Japan). Light sources are necessary for taking images in the wind tunnel. We selected them based on past experimental reports in JAXA.

- Marker: We attached markers for optical measurement to the model surface with dual layers. The first layer uses white seals with a diameter $\phi$ of $16 \mathrm{~mm}$; the second layer utilizes black transfer markers with $\phi$ of $8 \mathrm{~mm}$. Although the first layer is not always necessary, we used it to improve the recognition rate. As shown in Figure 4, we can explicitly detect the markers from the model image data.

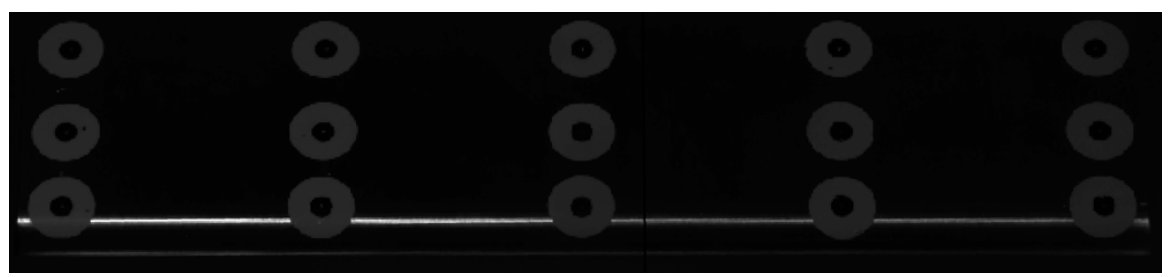

(a) Image data shot by the camera.

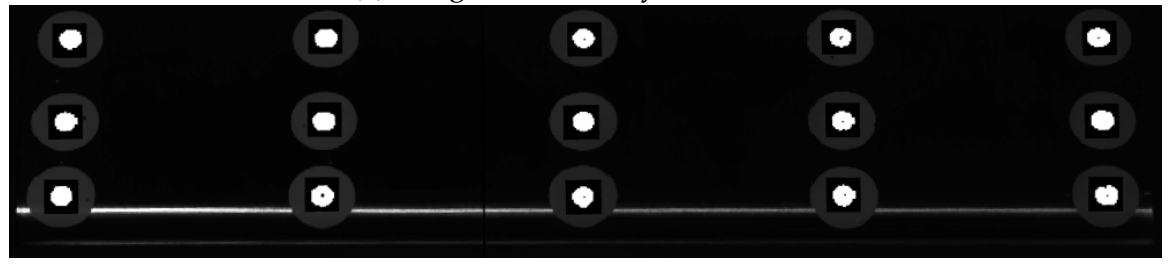

(b) Markers detected from an image's data.

Figure 4. Detection of markers on the flap upper surface.

\subsection{Instruments for Aerodynamic Measurement}

- Three-component force detector: We adopted LMC-3520A-500N made by NISSYO-ELECTRIC-WORKS CO., LTD. (Tokyo, Japan). A DC amplifier DSA-100B amplifies the voltage output from the detector; the secondary calibrator CAL-100-3B calibrates it. Both pieces of equipment are made by NISSYO-ELECTRIC-WORKS CO., LTD., too.

- Pressure scanner: We utilized an Intelligent Pressure Scanner 9016 made by Pressure Systems, Inc. (Hampton, VA, USA). 
- I/O device: We accepted two: NI USB-6501 to input encoder binary data and NI USB-6216BNC to output stepping motor pulse. National Instruments Co. (Austin, TX, USA) manufactures both devices.

\section{Performance Test Results for Remote and Feedback Control by Optical Measurement}

To assess the performance of the constructed system, we evaluated the tracking performance of the actuator by the difference $\Delta \theta_{\text {optical }}$ between input target angles $\theta_{\text {target }}$ and accomplished angles $\theta_{\text {optical }}$ on the flap:

$$
\Delta \theta_{\text {optical }}=\theta_{\text {optical }}-\theta_{\text {target }} \text {. }
$$

We always fixed the angle of attack of the main wing $\alpha$ at 0 degrees; we rotated the flap angle in the order of $0 \rightarrow-20 \rightarrow 0 \rightarrow 20 \rightarrow 0$ degrees. As shown in Figure 5, we defined the plus/minus of the rotation direction.

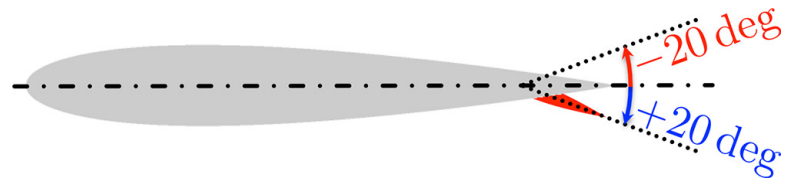

Figure 5. Definition of flap angle direction and operational range in performance tests for remote and open/feedback control.

We set $\theta_{\text {target }}$ to be eight angles: $-20,-10,-5,-1,1,5,10$, and 20 degrees to verify whether we control accurately regardless of the value of $\theta_{\text {target }}$. We performed under five wind speed conditions: 0 , $10,20,30,40$, and $50 \mathrm{~m} / \mathrm{s}$. We defined the converge judgment of this time as follows:

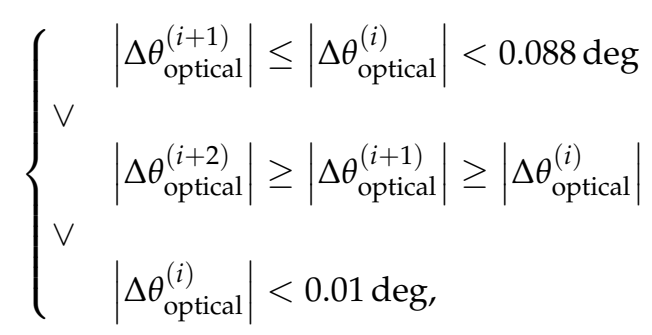

where $i(i=1,2,3, \cdots)$ signifies the iteration number of feedback controls. The second condition is a criterion for determining that the stepping motor is running idle.

In this case, we placed $\theta_{\text {target }}$ in the order of $0 \rightarrow-5 \rightarrow-10 \rightarrow-20 \rightarrow 0 \rightarrow 5 \rightarrow 10 \rightarrow 20 \rightarrow 0$ degrees. Figure 6a depicts final errors accomplished by the feedback control by $\Delta \theta_{\text {optical }}$ in each flap angle at each flow velocity. Except for a part of the data of $50 \mathrm{~m} / \mathrm{s}$ (when $\theta_{\text {target }}$ is $0 \mathrm{deg}$ ), $\Delta \theta_{\text {optical }}$ converged to

$$
\left|\Delta \theta_{\text {optical }}\right|<0.07 \mathrm{deg}
$$

Figure 6a reveals the following two points:

- The result in Figure 6a does not depend on any mechanical characteristics, such as the encoder resolution and the stepping motor's play. Thus, final errors by $\Delta \theta_{\text {optical }}$ are small.

- Several results succeeded in inhibiting $\Delta \theta_{\text {optical }}$ in the order of $\mathcal{O}\left(10^{-3}\right)$. If we arrange the current convergence criterion given by Equation (2) more strictly, final errors by $\Delta \theta_{\text {optical }}$ can decline further.

These data do not depend on wind speeds. Even under the $50 \mathrm{~m} / \mathrm{s}$ condition, the system achieved similar convergence performance except for $\theta_{\text {target }}$ of 0 degrees. In the case of 0 degrees, since the aerodynamic forces on the upper and lower surfaces of the flap are respectively small, they mutually cancel each other, and the feedback system cannot apply the torque against the aerodynamic force on 
the flap. Consequently, to lock the flap in the $\theta_{\text {target }}$ of 0 degrees is difficult, which is a drawback of the feedback control by $\Delta \theta_{\text {optical }}$.

Figure $6 \mathrm{~b}-\mathrm{g}$ shows the convergence histories of $\Delta \theta_{\text {optical }}$ for each wind speed. These graphs indicate the following three points:

- The controlled actuator speed is roughly

$$
\frac{\partial \Delta \theta_{\text {optical }}}{\partial t} \gtrsim 0.2 \mathrm{deg} / \mathrm{s}
$$

except for 40 and $50 \mathrm{~m} / \mathrm{s}$ wind velocity. Note that $t$ denotes the time required for control. Thus, a unit time of roughly at least $5.0 \mathrm{~s} /$ degrees is requisite for actuator rotations in the feedback control by $\Delta \theta_{\text {optical }}$. It takes time to control because we need the time to earn flap angles via optical measurement (obtaining the image with the cameras, detecting the markers, and computing flap angles).

- The feedback control by $\Delta \theta_{\text {optical }}$ rigorously measures the minute movement prompted by the system's play and the deformation of the wind tunnel model, so it takes long to converge using $\Delta \theta_{\text {optical }}$; the convergence of $\Delta \theta_{\text {optical }}$ becomes more troublesome as the wind speed is boosted. Since we cannot eliminate the mechanical play of the actuator, it is impossible in principle to make $\Delta \theta_{\text {optical }}$ zero.

- Due to the above reason, the number of times of control is inevitably necessary for positioning near $\theta_{\text {target. }}$ Thereupon, regardless of the wind speeds, the recurrence of the control by $\Delta \theta_{\text {optical }}$ rises. Moreover, the number of control times around $\theta_{\text {target }}$ abruptly grows under conditions at $40 \mathrm{~m} / \mathrm{s}$ or more flow velocities, but optical measurement technology does not induce this issue. As the wind velocity gains, the aerodynamic force matures stronger; the wind tunnel test model becomes liable to deform.

We needed many numbers of times of control and a control interval of up to $\mathcal{O}\left(10^{3}\right) \mathrm{s}$ because of the actuator mechanism and deformation of the wind tunnel test model, but we could control the flap even with the upper limit of the wind speed and could procure the flap angle data without any loss.

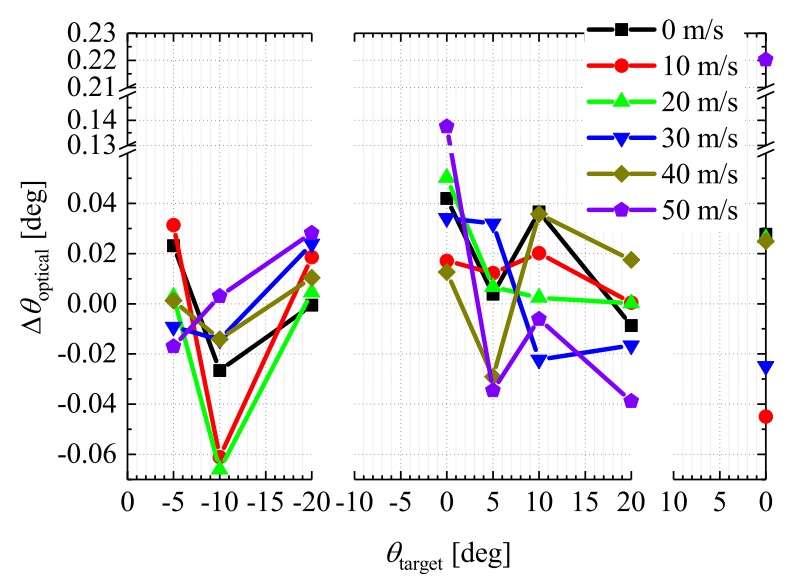

(a)

Figure 6. Cont. 


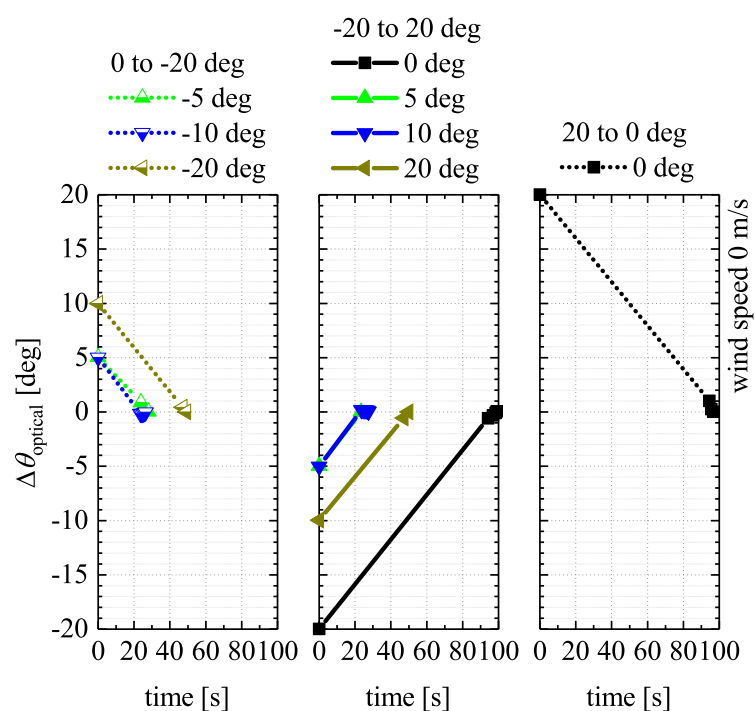

(b)

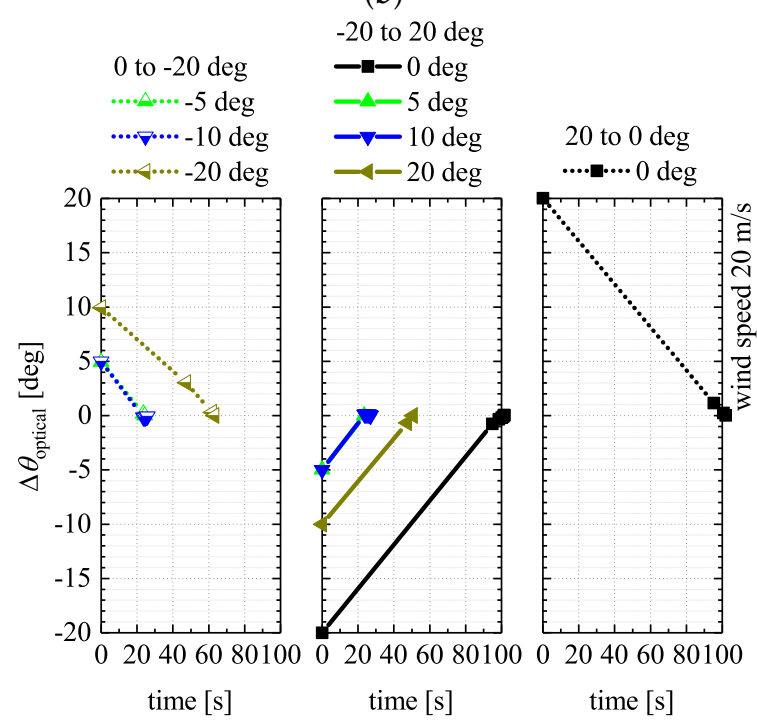

(d)

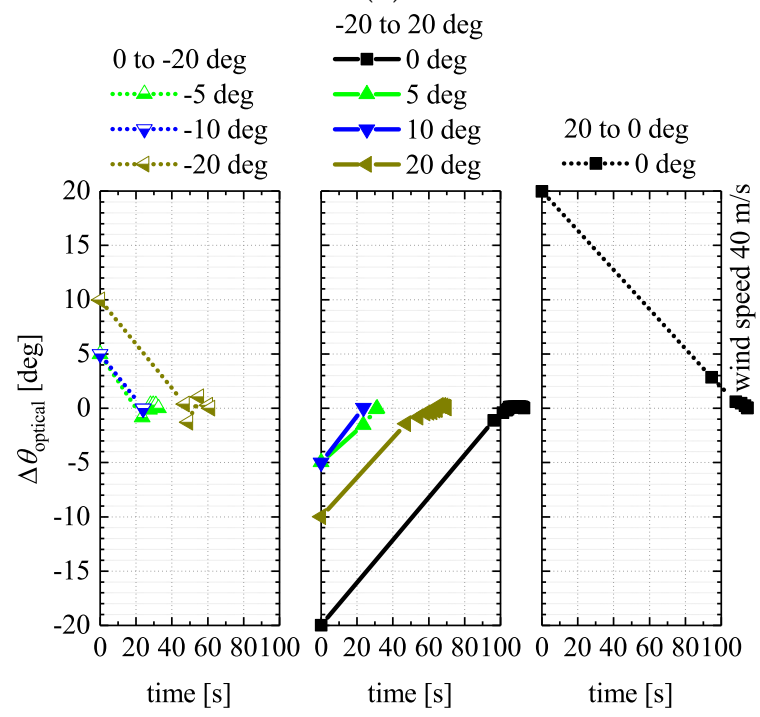

(f)

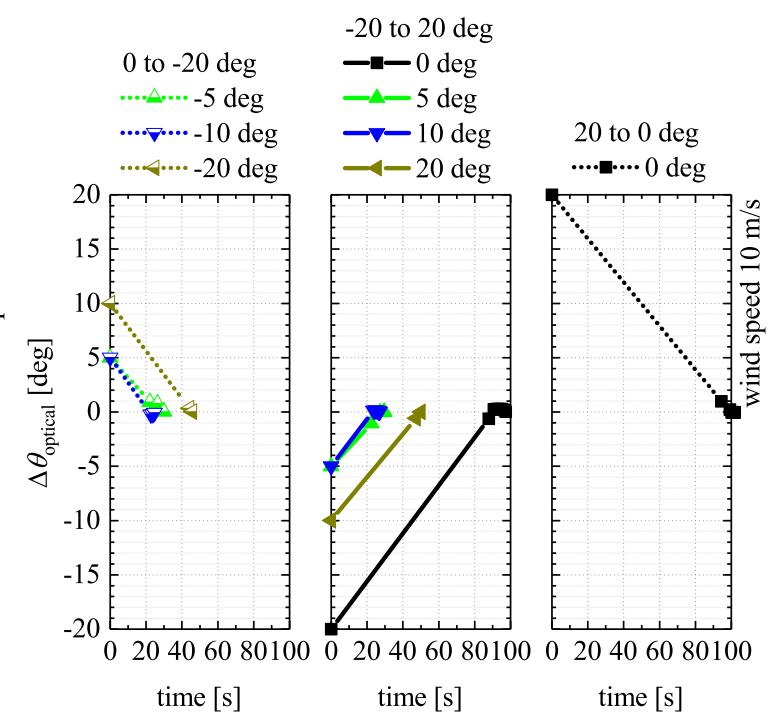

(c)

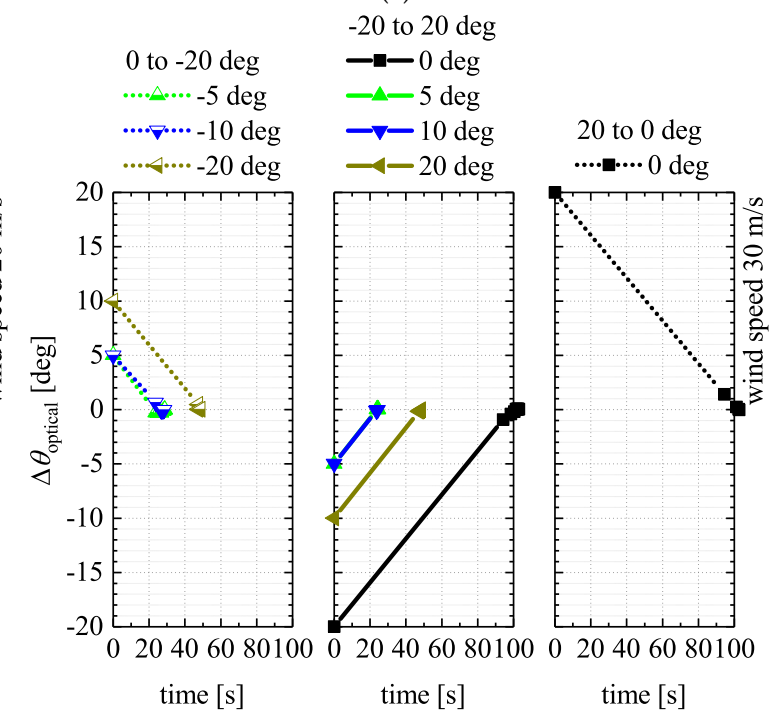

(e)

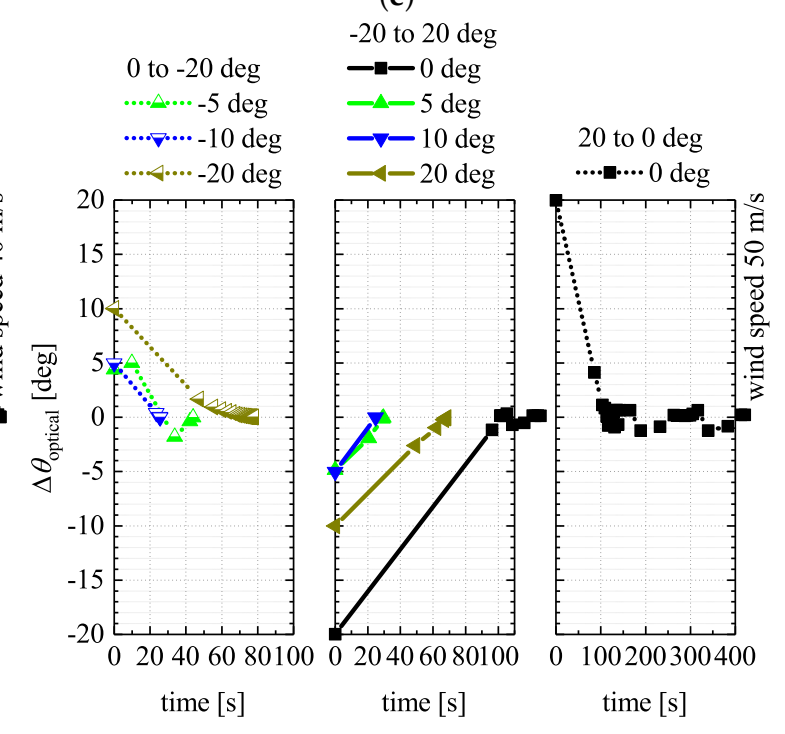

(g)

Figure 6. Results of the feedback control by $\Delta \theta_{\text {optical }}$. (a) $\Delta \theta_{\text {optical }}$ vs. $\theta_{\text {target }}$; each convergence history at wind speed of (b) 0, (c) 10, (d) 20, (e) 30, (f) 40, and (g) $50 \mathrm{~m} / \mathrm{s}$. 


\section{Conclusions}

This study has constructed a remote and feedback control system to adjust the configuration of a part of a wind tunnel test model. The purpose was to improve the efficiency of the wind tunnel test by automatically rotating the flap of the wind tunnel test model without pausing the ventilation and to position precisely by diverting optical measurement technology. We were able to experiment faithfully with the intended configurations because optical measurement can feedback for aerodynamic data from both the play of the actuator mechanism and the deformation of the model. Since sequential aerodynamic data could be procured efficiently by trial experiments presented in Appendix A, the system has expanded the possibility of future wind tunnel tests.

Author Contributions: K.C., T.K., H.K., and K.N. conceived and designed the experiments; T.K. and H.K. performed the experiments; K.C. and T.K. analyzed the data; K.C. wrote the paper. All authors have read and agreed to the published version of the manuscript.

Acknowledgments: This study was conducted under the JAXA student training system (technical acquisition).

Conflicts of Interest: The authors declare no conflict of interest.

\section{Appendix A. Attempt at Measuring Aerodynamic Three Coefficients}

Since we constructed the environment that accurately implements remote and feedback controls by optical measurement, we practically applied it to earn three components of the aerodynamic force. We calibrated three sensor components before and after the wind tunnel test. As shown in Figure A1, we fixed the model with one-side support mounted on a three-component force sensor on the bottom of the wind tunnel so that $25 \%$ mean aerodynamic chord was the moment center. We attempted experiments under the three conditions in Table A1. This endeavor is a feasibility study of acquiring aerodynamic data; the physical interpretation of the data will be our future work.

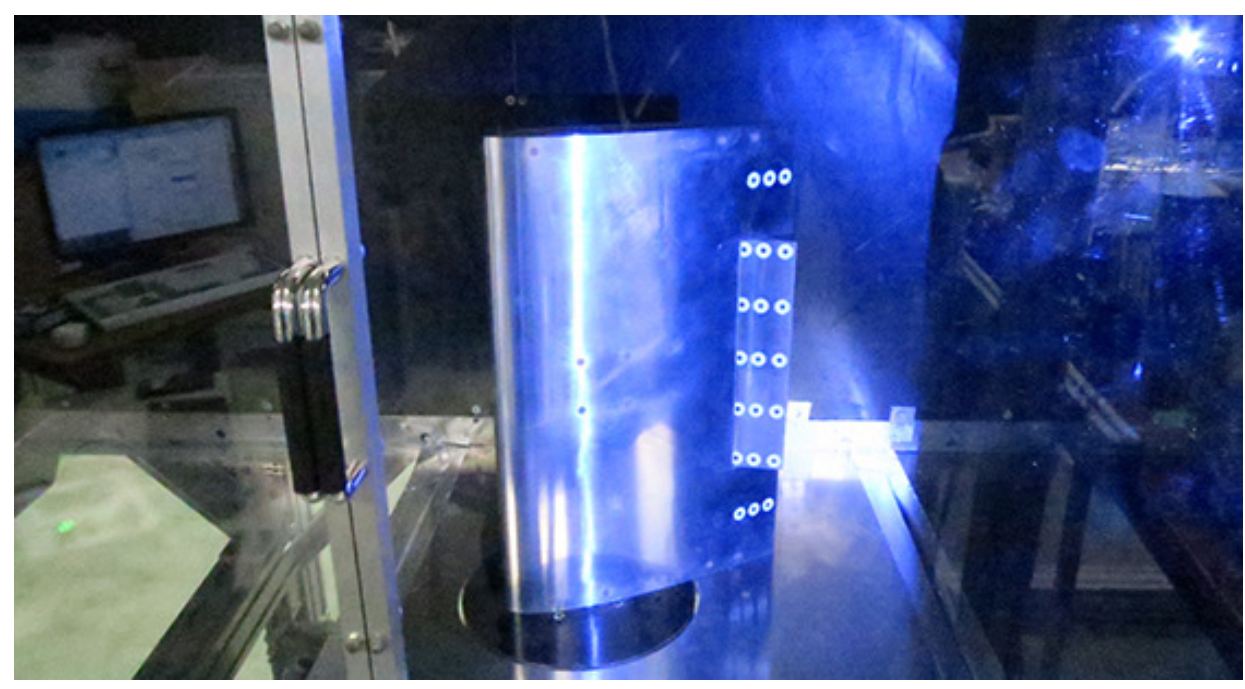

Figure A1. A state of aerodynamics measurement test.

Table A1. Experimental conditions for attempting aerodynamic measurement.

\begin{tabular}{rrrr}
\hline Case \# & Angle of Attack of the Main Wing $\boldsymbol{\alpha} \mathbf{( d e g )}$ & Wind Speed (m/s) & Sweep Range of the Flap (deg) \\
\hline 1 & 0 & 20 & from 0 to 40 \\
2 & 0 & 40 & from 0 to 40 \\
3 & 21 & 20 & from -40 to 45 \\
\hline
\end{tabular}

Figure A2a presents the gained $C_{L}$ and $C_{D}$; Figure A2b displays $C_{M p}$. Since Cases 1 and 2 were system operation confirmation works, we measured $C_{L}$ and $C_{D}$ only in Case 3 . Figure A2 explicates the state in which the experiments could obtain aerodynamic data sequentially. From the results of 
Cases 1 and 2, the contrast due to the wind speed is also apparent; the system accomplished high resolution that the gradient $\partial C_{M_{p}} / \partial \theta_{\text {target }}$ changes near $\theta_{\text {target }}=13$ degrees in Case 1 . It is significant to be able to capture nonlinear aerodynamic change due to an alteration in the flow around the flap.

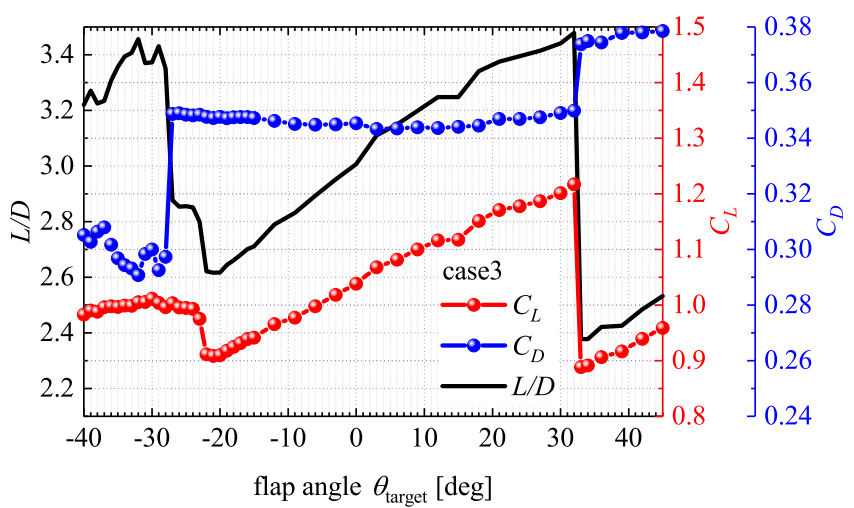

(a) $C_{L}, C_{D}$, and $L / D$

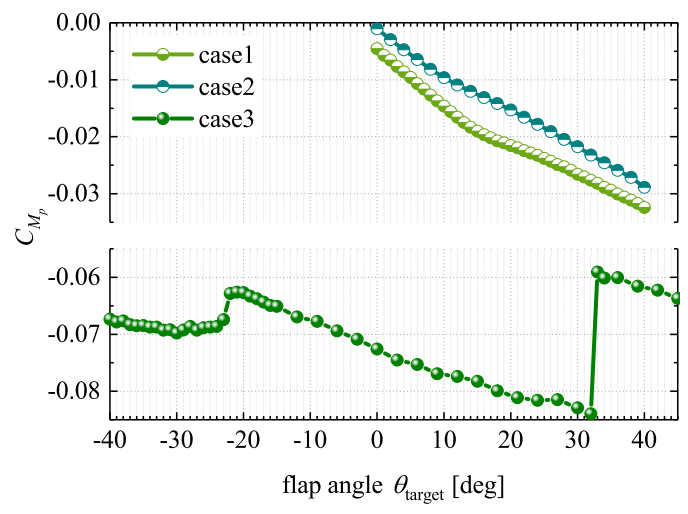

(b) $C_{M_{p}}$

Figure A2. Three aerodynamic coefficients earned by the feedback control with optical measurement.

Next, we plan to perceive the behavior of the data of Case 3 that possesses three components $\left(C_{L}\right.$, $C_{D}$, and $C_{M}$ ) under the condition that the main wing is at the high angle of attack. Figure A2 indicates that phenomena vary between the four regions: (i) $-40 \mathrm{deg} \leq \theta_{\text {target }} \leq-27 \mathrm{deg}$, (ii) $-26 \mathrm{deg} \leq$ $\theta_{\text {target }}<-23 \mathrm{deg}$, (iii) $-22 \mathrm{deg} \leq \theta_{\text {target }} \leq 32 \mathrm{deg}$, and (iv) $33 \mathrm{deg} \leq \theta_{\text {target }} \leq 45 \mathrm{deg}$. Visualizing flow fields requires comprehending specific physics, but we infer phenomena as follows:

(i) A separation that occurs on the main wing upper surface interferes with the flap.

(ii) Since the flap is lying down, it entirely goes inside the separation that arises on the main wing.

(iii) Since we set 21 degrees for the angle of attack of the main wing, the flap is horizontal at $\theta_{\text {target }}$ of -21 degrees. $\theta_{\text {target }}$ over 21 degrees commences activating the flap; the lift gains linearly.

(iv) The flap works as a spoiler because the flap is 54 degrees or more for the uniform stream.

In this way, the system can sequentially capture variations in phenomena in one ventilation run; we can precisely distinguish changing physical circumstances.

In this endeavor, $\theta_{\text {target }}$ rotated from -40 to 45 degrees, but by performing the reverse rotation, we can address a hysteresis of aerodynamic force. In addition to facilitating the capabilities of wind tunnel tests, we would perceive fluid physics more deeply hereafter.

\section{References}

1. van Dam, C.P. The Aerodynamic Design of Multi-Element High-Lift Systems for Transport Airplanes. Prog. Aerosp. Sci. 2002, 38, 101-144. [CrossRef]

2. Rogers, M.M. Progress towards the CFD Vision 2030; NASA Technical Reports ARC-E-DAA-TN60442; NASA: Washington, DC, USA, 2018.

3. Mavriplis, D.J. Progress in CFD Discretizations, Algorithms and Solvers for Aerodynamic Flows; AIAA Paper 2019-2944 on the AIAA Aviation 2019 Forum; AIAA: Reston, VA, USA, 2019. [CrossRef]

4. Chawner, J.R.; Taylor, N.J. Progress in Geometry Modeling and Mesh Generation toward the CFD Vision 2030; AIAA Paper 2019-2945 on the AIAA Aviation 2019 Forum; AIAA: Reston, VA, USA, 2019. [CrossRef]

5. Rumsey, C.L.; Slotnick, J.P.; Long, M.; Stuever, R.A.; Wayman, T.R. Summary of the First AIAA CFD High-Lift Prediction Workshop. J. Aircr. 2011, 48, 2068-2079. [CrossRef]

6. Rumsey, C.L.; Slotnick, J.P. Overview and Summary of the Second AIAA High-Lift Prediction Workshop. J. Aircr. 2015, 52, 1006-1025. [CrossRef]

7. Rumsey, C.L.; Slotnick, J.P.; Sclafani, A.J. Overview and Summary of the Third AIAA High-Lift Prediction Workshop. J. Aircr. 2018, 56, 621-644. [CrossRef] 
8. Adrian, R.J. Particle-Imaging Techniques for Experimental Fluid Mechanics. Ann. Rev. Fluid Mech. 1991, 23, 261-304. [CrossRef]

9. McLachlan, B.G.; Bell, J.H. Pressure-Sensitive Paint in Aerodynamic Testing. Exp. Therm. Fluid Sci. 1995, 10, 470-485. [CrossRef]

10. Gregory, J.W.; Asai, K.; Kameda, M.; Liu, T.; Sullivan, J.P. A Review of Pressure-Sensitive Paint for High-Speed and Unsteady Aerodynamics. Proc. Inst. Mech. Eng. Part G J. Aerosp. Eng. 2008, 222, 249-290. [CrossRef]

11. Bergmann, A.; Huebner, A.; Loeser, T. Experimental and Numerical Research on the Aerodynamics of Unsteady Moving Aircraft. Prog. Aerosp. Sci. 2008, 44, 121-137. [CrossRef]

12. Bergmann, A. Modern Wind Tunnel Techniques for Unsteady Testing-Development of Dynamic Test Rigs. In Hermann Schlichting-100 Years. Notes on Numerical Fluid Mechanics and Multidisciplinary Design; Springer: Berlin/Heidelberg, Germany, 2009; Volume 102, pp. 59-77. [CrossRef]

13. Ulrich, A.; Akkerman, E.; Kotschote, J.; Villa, A. Remotely Controlled Movable Surface Motorization of an Industrial Used Wind Tunnel Model; AIAA Paper 2010-4338 on the 27th AIAA Aerodynamic Measurement Technology and Ground Testing Conference; AIAA: Reston, VA, USA, 2010.

14. Smith, C.A. Wind Tunnel Model Measuring System and Method. US 8,783,098 B2, 22 July 2014.

15. Ternoy, F.; Dandois, J.; David, F.; Pruvost, M. Overview of Onera Actuators for Active Flow Control. AerospaceLab 2013, 6, 1-14.

16. Asai, K.; Amao, Y.; Iijima, Y.; Okura, I.; Nishide, H. Novel Pressure-Sensitive Paint for Cryogenic and Unsteady Wind-Tunnel Testing. J. Thermophys. Heat Transf. 2002, 16, 109-115. [CrossRef]

17. Nakakita, K.; Kurita, M.; Mitsuo, K.; Watanabe, S. Practical pressure-sensitive paint measurement system for industrial wind tunnels at JAXA. Meas. Sci. Technol. 2006, 17, 359-366. [CrossRef]

18. Watanabe, S.; Kato, H.; Kwak, D.Y.; Shirotake, M.; Rinoie, K. Stereoscopic PIV measurements of leading edge separation vortices on a cranked arrow wing. Meas. Sci. Technol. 2004, 15, 1079-1089. [CrossRef]

19. Kato, H.; Koike, S.; Nakakita, K. Time-Resolved Stereoscopic PIV Measurement of Unsteady Wingtip Flowfield; AIAA Paper 2012-0035; AIAA: Reston, VA, USA, 2012. [CrossRef]

20. Nakakita, K. Simultaneous Visualization of Transonic Buffet on a Rocket Faring Model Using Unsteady PSP Measurement and Schlieren Method. In Proceedings of the The 31st International Congress on High-Speed Imaging and Photonics, Osaka, Japan, 7-10 November 2016. [CrossRef]

(C) 2020 by the authors. Licensee MDPI, Basel, Switzerland. This article is an open access article distributed under the terms and conditions of the Creative Commons Attribution (CC BY) license (http:/ / creativecommons.org/licenses/by/4.0/). 